Research Article

\title{
The Effect of Lower-Limb Exercise on Pain Management of the Patients Undergoing Posterior Lumbar Fusion Surgery: A Retrospective Case-Control Study
}

\author{
Tong $\mathrm{Wu}$ (iD) ${ }^{1,2}$ and Yong $\mathrm{Ye}^{3}$ \\ ${ }^{1}$ Department of Orthopedic Surgery, Nantong Tongzhou People's Hospital, 115 Jianshe Road, 226300 Nantong, Jiangsu, China \\ ${ }^{2}$ Department of Orthopedic Surgery, Nantong University Affiliated Tongzhou Hospital, 115 Jianshe Road, 226300 Nantong, \\ Jiangsu, China \\ ${ }^{3}$ Department of Orthopedic Surgery, Wenzhou Central Hospital, 252 Baili East Road, 325000 Wenzhou, Zhejiang, China
}

Correspondence should be addressed to Tong Wu; 1816320129@yjs.ntu.edu.cn

Received 23 October 2021; Accepted 19 November 2021; Published 3 December 2021

Academic Editor: Sidong Yang

Copyright (c) 2021 Tong Wu and Yong Ye. This is an open access article distributed under the Creative Commons Attribution License, which permits unrestricted use, distribution, and reproduction in any medium, provided the original work is properly cited.

\begin{abstract}
Purpose. The purpose of this study is to investigate the clinical effect of lower-limb exercise, when combined with celecoxib, on pain management of patients undergoing posterior lumbar fusion surgeries. Methods. The patients undergoing posterior lumbar fusion surgeries between 01/2018 and 06/2021 were retrospectively identified, with their data collected. After surgery, some patients took celecoxib for analgesia (celecoxib group, $200 \mathrm{mg} /$ day) while the others took celecoxib together with lower-limb exercise (combined group, celecoxib-200 mg/day). On postoperative days (POD) 1, 3, 7, and 14, data were collected and analyzed regarding the following items: patient satisfaction, lower-limb muscle force, lumbar JOA score (29 points), Oswestry Disability Index (ODI), and visual analog scale (VAS) score. Results. A total of 225 participants were included in this study. Specifically, 120 cases were admitted into in the celecoxib group and 105 were included in the combined group. Comparisons of baseline data did not indicate any difference between the combined group and the celecoxib group. Data analysis showed that patient satisfaction in the combined group was significantly higher than the celecoxib group on POD 3, 7, and 14, respectively (all $p<0.001$ ). Moreover, the combined group had less VAS score compared with the celecoxib group on POD 3, 7, and 14, respectively (all $p<0.01$ ). In addition, lower-limb muscle force in the combined group was significantly stronger than that in the celecoxib group on POD 3 and POD 7, respectively (both $p<0.01$ ). Furthermore, the combined group achieved less ODI score than the celecoxib group on POD 3,7 , and 14, respectively (all $p<0.05$ ). Comparisons of the lumbar JOA score did not suggest any statistical difference during the whole follow-up period. Conclusions. In conclusion, postoperative lower-limb rehabilitation exercise can help to release pain after lumbar fusion surgeries. Additionally, postoperative lower-limb exercise can facilitate the recovery of lower-limb muscle force, as well as improving patient satisfaction.
\end{abstract}

\section{Introduction}

In clinical scenarios, lower back pain (LBP) mainly derives from intervertebral disc degeneration (IVDD) [1-3]. IVDD-related diseases, such as lumbar disc herniation, can lead to severe symptoms including LBP and lower-limb radicular pain. In such a situation, the patients usually need to undergo surgeries to remove the protruded disc and decompress the nerve root and spinal canal. To date, posterior lumbar interbody fusion surgery has been a widely used surgical procedure for treating IVDD-derived diseases, in particular, lumbar spinal diseases [4-6]. However, it has been reported by previous studies that postoperative patients may experience prolonged LBP and low quality of life [7-10], that is one of the key reasons that some patients would seek physical therapy after lumbar fusion surgery, for a purpose of speeding up their rehabilitation. 
As suggested by previous studies, postoperative rehabilitation procedures can help pain management after spinal surgeries, even decreasing the disability events [11-13]. In the past few years, accumulative studies have indicated that postoperative lower-limb exercise can facilitate rehabilitation and help relieve pain after orthopedic surgery [14] and lumbar spine surgery $[15,16]$. Clinically, celecoxib, one of nonsteroidal anti-inflammatory drugs (NSAIDS), has been commonly administered to the patients for pain relief, the regular oral dose of celecoxib being $200 \mathrm{mg}$ daily [17-19]. However, it remains unclear whether postoperative lower-limb exercise can increase pain relief when administered together with celecoxib for the patients undergoing spine surgery.

Thus, the purpose of this study is to investigate the effect of lower-limb exercise, when combined with celecoxib, on pain management of the patients who undergo posterior lumbar fusion surgeries.

\section{Patients and Methods}

2.1. Ethics. Prior to the commencement of this study, the medical ethics has been approved by Medical Ethics Council of Nantong Tongzhou People's Hospital. All informed consent was signed by the patients (or their lawful guardians) before undergoing lumbar fusion surgeries.

2.2. Patients. The patients undergoing posterior lumbar fusion surgeries (Figure 1) between 01/2018 and 06/2021 were retrospectively identified and screened. All participants were diagnosed with lumbar disc herniation or lumbar spinal stenosis. The related data were then collected, including the data followed up with different time points on postoperative days (POD) 1, 3, 7, and 14. After surgery, some of these patients took celecoxib for analgesia (celecoxib group, celecoxib$200 \mathrm{mg}$ /day), while the others took celecoxib together with lower-limb exercise (combined group, celecoxib-200 mg/day). Both in the celecoxib group and the combined group, celecoxib was administrated to the patients $200 \mathrm{mg} /$ time/day, administrated in the evening. During the perioperation period, all the participants underwent the same routine medical care regardless of treatment groups. Postoperatively, the patients in the combined group did rehabilitation exercise by following the lower-limb rehabilitation procedures as previously reported [14-16] and maintained for up to 14 days.

2.3. Assessment. Data were collected and analyzed regarding the following items: patient satisfaction, lower-limb muscle force, lumbar JOA score (29 points), Oswestry Disability Index (ODI), and visual analog scale (VAS) score. The grading of lower-limb muscle force was based on the classification criteria which British Medical Research Council applies. In addition, the patient satisfaction rate was scored to three levels: very satisfied, satisfied, and dissatisfied.

2.4. Statistical Analyses. Statistical analysis in this study was performed using the software SPSS for Windows 18.0 (SPSS Inc., USA). The data of ODI, JOA score, and VAS score are presented as mean \pm standard deviation (SD). The data of age are presented as median (range). Multiple comparisons were carried out with analysis of variance (ANOVA) if homogeneity and normality of variance were assumed, subsequently followed by Student-Newman-Keuls $t$-tests used to identify the difference between two groups. Moreover, chisquare tests were conducted to analyze the categorical data (gender, patient satisfaction, and muscle force). A $p$ value of 0.05 was set as the significance level.

\section{Results}

3.1. Baseline Data of Participants. After the identification of all patients, a total of 225 participants were included in this case-control study. Specifically, 120 cases were admitted into the celecoxib group and 105 cases were included in the combined group. The combined group consists of 48 males and 57 females, while the celecoxib group consists of 54 males and 66 females. The median age of the combined group is 54 years (range 21-67), while the median age of the celecoxib group is 56 years (range 23-70). Comparisons of those baseline data did not suggest any difference between the combined group and the celecoxib group (all $p>0.05$ ).

3.2. Patient Satisfaction. As given in Table 1, the patient satisfaction was categorized to three grades: very satisfied, satisfied, and dissatisfied. Most of the patients were very satisfied and satisfied about their treatment regardless of the treatment groups or postoperative time points. Data analysis showed that patient satisfaction in the combined group was significantly higher than the celecoxib group on POD 3,7 , and 14 , respectively (all $p<0.001$ ). There was no difference regarding the patient satisfaction on POD 1 between the combined group and the celecoxib group $(p>0.05)$.

3.3. VAS Score. As given in Table 2, the combined group obtained less VAS score compared with the celecoxib group on $\mathrm{POD} 3(2.6 \pm 1.2$ vs. $3.5 \pm 1.1)$, POD $7(1.5 \pm 1.2$ vs. $2.3 \pm 1.1)$, and POD $14(1.1 \pm 0.3$ vs. $1.2 \pm 0.2)$, respectively (all $p<0.01$ ). No significant difference of VAS score was indicated on POD 1 between the combined group and the celecoxib group $(p>0.05)$.

3.4. Lower-Limb Muscle Strength Grading. As given in Table 3, most grading of the preoperative lower-limb muscle strength was grade III and grade IV in both the combined group and the celecoxib group. Grade IV and grade V (as a whole) took the majority after surgery, and grade $\mathrm{V}$ increased continuously during the postoperative follow-up period regardless of the groups. As compared with the preoperative grading, the muscle strength got improved in both the combined group and the celecoxib group. Lowerlimb muscle force in the combined group was significantly stronger than that in the celecoxib group on POD 3 and POD 7 , respectively (both $p<0.01$ ). There was no significant difference found between the combined group and the celecoxib group on POD 1 or POD 14. 


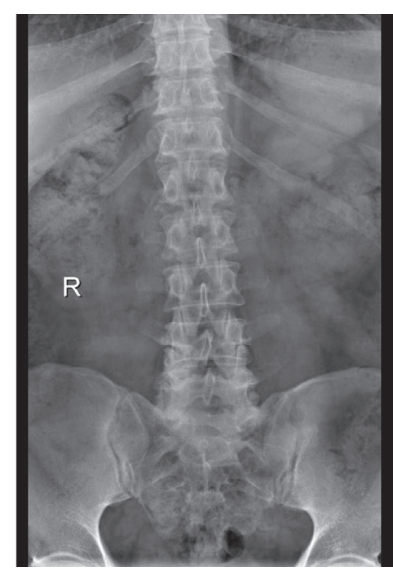

(a)

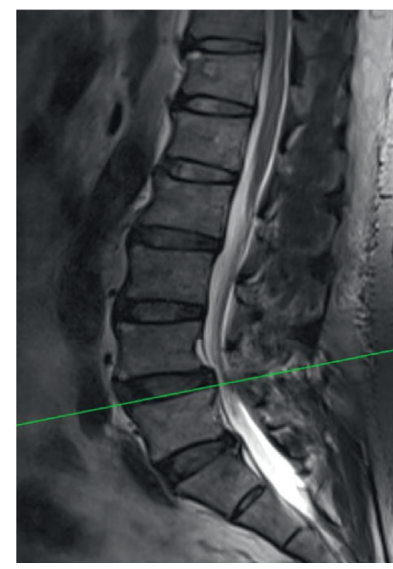

(e)

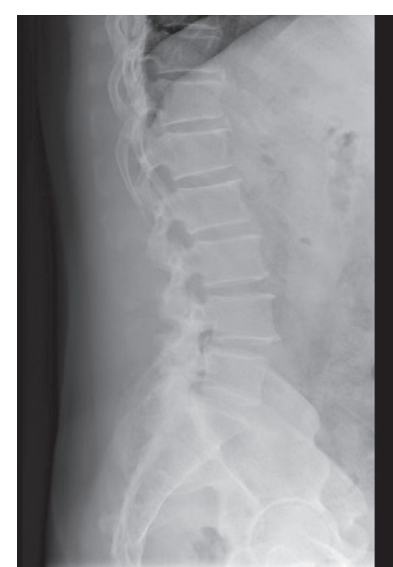

(b)

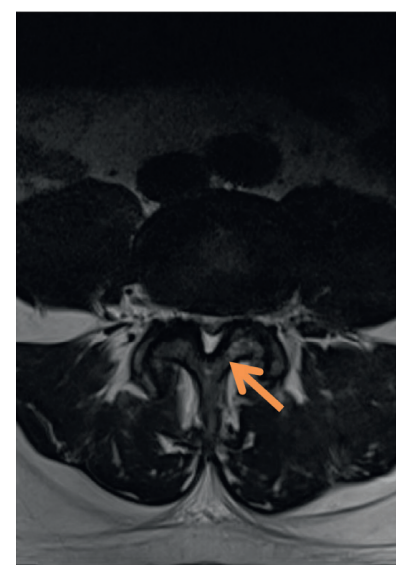

(f)

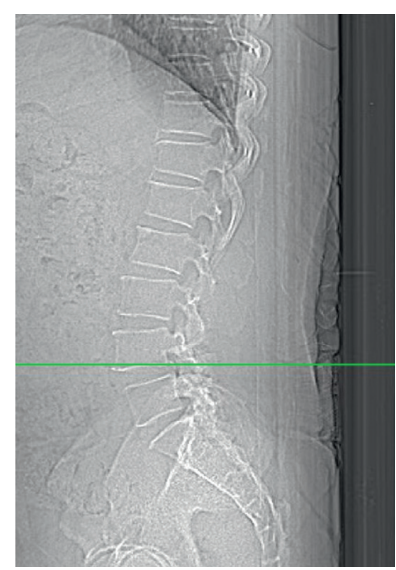

(c)

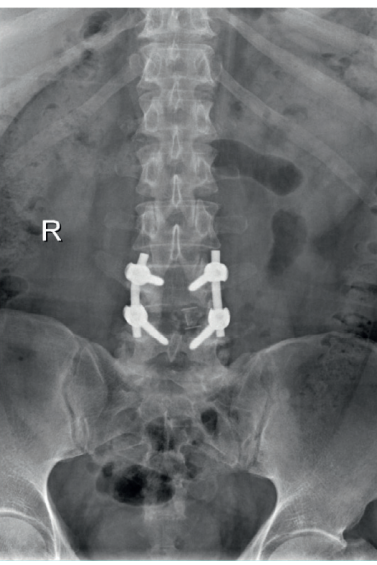

$(\mathrm{g})$

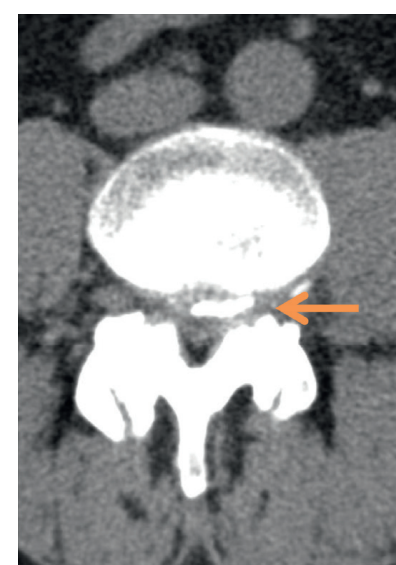

(d)

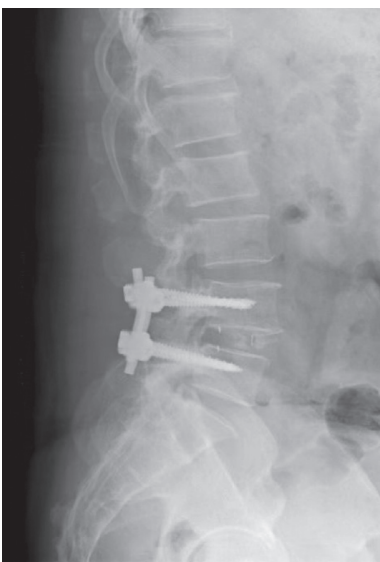

(h)

Figure 1: A representative case of posterior fusion surgery. (a)-(b) Preoperative X-ray radiographs. (c)-(d) Preoperative CT scan. (e)-(f) Preoperative MRI scan. (g)-(h) Postoperative X-ray radiographs. The arrows indicate the herniation of nucleus pulposus in the intervertebral disc.

TABle 1: Patient satisfaction at discharge.

\begin{tabular}{|c|c|c|c|c|}
\hline \multirow{2}{*}{ After surgery } & \multirow{2}{*}{$\begin{array}{l}\text { Combined group }(n=105) \\
\text { Very satisfied/satisfied/dissatisfied }\end{array}$} & \multirow{2}{*}{$\begin{array}{l}\text { Celecoxib group }(n=120) \\
\text { Very satisfied/satisfied/dissatisfied }\end{array}$} & \multicolumn{2}{|c|}{ Chi-square tests } \\
\hline & & & $\chi^{2}$ & $P$ value \\
\hline POD 1 & 30 cases $/ 60$ cases $/ 15$ cases & 32 cases $/ 69$ cases $/ 19$ cases & 0.164 & 0.921 \\
\hline POD 3 & 72 cases $/ 30$ cases $/ 3$ cases & 40 cases $/ 64$ cases $/ 16$ cases & 29.47 & $<0.001$ \\
\hline POD 7 & 92 cases $/ 12$ cases $/ 1$ cases & 52 cases $/ 58$ cases $/ 10$ cases & 47.92 & $<0.001$ \\
\hline POD 14 & 101 cases $/ 4$ cases $/ 0$ case & 78 cases $/ 37$ cases $/ 5$ cases & 33.67 & $<0.001$ \\
\hline
\end{tabular}

All values with $\mathrm{P}<0.05$ are presented in bold, which indicate statistical significance. Combined group, celecoxib and lower-limb rehabilitation exercise; POD, postoperative day.

TABLE 2: Assessment of VAS score.

\begin{tabular}{|c|c|c|c|c|c|}
\hline Group & Pre-op & POD 1 & POD 3 & POD 7 & POD 14 \\
\hline Celecoxib $(n=120)$ & $6.8 \pm 1.7$ & $5.7 \pm 1.4$ & $3.5 \pm 1.1$ & $2.3 \pm 1.1$ & $1.2 \pm 0.2$ \\
\hline Combined $(n=105)$ & $6.6 \pm 2.1$ & $5.4 \pm 1.6$ & $2.6 \pm 1.2$ & $1.5 \pm 1.2$ & $1.1 \pm 0.3$ \\
\hline$P$ value & 0.431 & 0.135 & $<0.001$ & $<0.001$ & 0.003 \\
\hline
\end{tabular}

All values with $\mathrm{P}<0.05$ are presented in bold, which indicate statistical significance.VAS, visual analog scale; combined group, celecoxib and lower-limb rehabilitation exercise; POD, postoperative day; Pre-op, preoperation.

3.5. ODI Score. As given in Table 4, preoperatively, there was no significant difference regarding the ODI score between the combined group and the celecoxib group $(p>0.05)$. Postoperatively, both of the combined group and the celecoxib group achieved significant improvement of the ODI score, compared to their preoperative data, respectively. The combined group scored less ODI than the celecoxib group on POD 3,7 , and 14 , respectively (all $(p<0.05)$ ). There was no statistical difference between the combined group and the celecoxib group on POD $1(p>0.01)$. 
TABLE 3: Lower-limb muscle force.

\begin{tabular}{|c|c|c|c|c|c|c|c|c|c|c|c|c|c|c|c|}
\hline Group & & Pre-op & & & POD 1 & & & POD 3 & & & POD 7 & & & POD 14 & \\
\hline Grade & III & IV & $\mathrm{V}$ & III & IV & $\mathrm{V}$ & III & IV & $\mathrm{V}$ & III & IV & $\mathrm{V}$ & III & IV & $\mathrm{V}$ \\
\hline Celecoxib $(n=120)$ & 34 & 68 & 18 & 31 & 77 & 12 & 28 & 75 & 17 & 25 & 64 & 31 & 12 & 24 & 84 \\
\hline Combined $(n=105)$ & 27 & 63 & 15 & 23 & 72 & 10 & 18 & 52 & 35 & 12 & 43 & 50 & 8 & 18 & 79 \\
\hline $\begin{array}{l}\chi^{2} \\
P \text { value }\end{array}$ & & $\begin{array}{c}0.268 \\
P=0.875\end{array}$ & & & $\begin{array}{c}0.537 \\
P=0.764\end{array}$ & & & $\begin{array}{c}11.622 \\
P=0.003\end{array}$ & & & $\begin{array}{c}12.200 \\
P=0.002\end{array}$ & & & $\begin{array}{c}0.814 \\
P=0.666\end{array}$ & \\
\hline
\end{tabular}

$P<0.001$, in terms of muscle force comparison between the celecoxib group and the combined group. POD, postoperative day; combined group, celecoxib and lower-limb rehabilitation exercise; Pre-op, preoperation.

TABLE 4: ODI assessment and comparisons.

\begin{tabular}{lccccc}
\hline Groups & Pre-op & POD 1 & POD 3 & POD 7 & POD 14 \\
\hline Celecoxib $(n=120)$ & $48 \pm 22$ & $41 \pm 20$ & $33 \pm 16$ & $21 \pm 11$ & $13 \pm 4$ \\
Combined $(n=105)$ & $46 \pm 23$ & $40 \pm 19$ & $27 \pm 15$ & $18 \pm 9$ & $11 \pm 4$ \\
$P$ value & 0.506 & 0.702 & $\mathbf{0 . 0 0 4 2}$ & $\mathbf{0 . 0 2 7}$ & $<\mathbf{0 . 0 0 1}$
\end{tabular}

All values with $\mathrm{P}<0.05$ are presented in bold, which indicate statistical significance.ODI, Oswestry Disability Index; combined group, celecoxib and lower-limb rehabilitation exercise; POD, postoperative day; Pre-op, preoperation.

3.6. Lumbar JOA Score. As given in Table 5, preoperatively, there was no significant difference regarding the lumbar JOA score between the combined group and the celecoxib group $(p>0.05)$. Postoperatively, comparisons of the lumbar JOA score did not suggest any statistical differences between the combined group and the celecoxib group during the whole follow-up period $(p>0.05)$.

\section{Discussion}

In our department, as a routine procedure for the prophylaxis of potential postoperative complications (such as deep vein thrombosis), the patients are required to do lower-limb rehabilitation exercise postoperatively; the exercise procedures are given in previous studies [14-16]. All participants are asked to do the same intensity rehabilitation for up to two weeks. The pain relief effect of lower-limb rehabilitation exercise on the patients after orthopedic surgery and spinal surgery has already been documented in those previous studies [14-16]. In this study, the patients in the combined group kept doing rehabilitation exercise for up to 14 days. That was because many patients can walk well and start to do some normal exercise, other than the lower-limb rehabilitation procedures, after 14 days after surgery. Thus, 14-day exercise with the lower-limb rehabilitation procedures after surgery was considered as an endpoint of our study on the postoperative pain management.

Celecoxib, a selective cyclooxygenase- 2 inhibitor and an NSAID, has been routinely used by the patients after spinal surgery for pain relief, with its advantage of minimizing the gastrointestinal adverse effects [17]. Considering the pain relief effects of celecoxib and lower-limb exercise, it would be possible for them to have synergistic effects on pain relief. However, thus far, it has been unclear whether postoperative lower-limb exercise can increase pain relief when administered together with celecoxib for the patients undergoing
TABle 5: JOA score (lumbar, 29 points) assessment and comparisons.

\begin{tabular}{lccccc}
\hline Groups & Pre-op & POD 1 & POD 3 & POD 7 & POD 14 \\
\hline $\begin{array}{l}\text { Celecoxib } \\
(n=120)\end{array}$ & $7.5 \pm 1.3$ & $10.5 \pm 1.5$ & $14.7 \pm 2.2$ & $19.8 \pm 6.1$ & $22.1 \pm 6.6$ \\
$\begin{array}{l}\text { Combined } \\
(n=105)\end{array}$ & $7.8 \pm 1.2$ & $10.2 \pm 1.7$ & $15.1 \pm 2.4$ & $20.3 \pm 6.3$ & $22.3 \pm 6.5$ \\
$P$ value & 0.075 & 0.161 & 0.194 & 0.546 & 0.820 \\
\hline
\end{tabular}

JOA, Japanese Orthopedic Association; combined group, celecoxib and lower-limb rehabilitation exercise; POD, postoperative day; Pre-op, preoperation.

spine surgery. Thus, this study was designed to investigate the effect of lower-limb exercise, when combined with celecoxib, on pain management of the patients who underwent posterior lumbar fusion surgery in our department.

As a result, a total of 225 cases were included in our study. Baseline data (age and gender) were well matched between the combined group and the celecoxib group. Compared with preoperative situations, the combined group and the celecoxib group have significantly improved in terms of the patient satisfaction, VAS score, lower-limb muscle force, lumbar JOA score, and ODI score. Also, it was found that the combined group achieved better results than the celecoxib group, in terms of the patient satisfaction, VAS score, lower-limb muscle force, and ODI score. These findings in this study stay consistent with the reports from previous studies [14-16] which indicate that lower-limb exercise can effectively increase postoperative pain relief, accelerate functional recovery, and decrease complications (such as deep vein thrombosis).

It is noticeable in this study that the postoperative lumbar JOA score is not significantly different between the combined group and the celecoxib group during the whole follow-up period (up to 14 days). This result of the JOA score is inconsistent with previous reports indicating that postoperative lower-limb exercise can improve the JOA score. One possible reason for this result is that our follow-up period is too short, only 14 days postsurgery. By contrast, the maximum follow-up period in the previous studies are up to 3 months [14-16]. Another reason could be the different study designs between this study and other studies. In this study, the combined group was designed to compare with the celecoxib group, while the lower-limb exercise group was compared with the control group (settings unknown) in those previous studies.

Up to now, there is no consensus regarding whether postoperative rehabilitation can effectively promote the 
recovery of patients undergoing spinal surgery. In terms of pain relief, functional improvement, and patient satisfaction, the positive effects of postoperative rehabilitation procedures have been declared in some studies [12, 14, 16, 20, 21], while some others are negative towards postoperative rehabilitation $[4,22,23]$. Apparently, the findings in the current study support the former, with increased pain relief, great functional improvement, and higher patient satisfaction in the rehabilitation group, compared to the nonrehabilitation group postoperatively.

This study has some limitations that might have restricted the interpretation of the data. First, this is a singlecenter, retrospective, case-control study, making the participants included lack for extensive representativeness and the data accuracy decreases to a certain extent. In addition, the patient sample is not large, just a total of 225 participants were included in this study. It would make the results and conclusions more robust if the patient sample size is greater. Moreover, the follow-up period in this study is not long enough (just 14 days), which can potentially influence the results and conclusions. Therefore, a better future study needs to resolve all of the shortcomings listed above. It can be designed to be multicenter, prospective, blinded, and randomly controlled; the sample size should be big enough.

\section{Conclusions}

In summary, postoperative lower-limb rehabilitation exercise can synergistically work with celecoxib, increasing pain relief for the patients undergoing lumbar fusion surgeries. In addition, postoperative lower-limb exercise can facilitate functional recovery and increase patient satisfaction.

\section{Data Availability}

The data used to support the findings of this study are available from the corresponding author upon request.

\section{Conflicts of Interest}

The authors declare that they have no conflicts of interest.

\section{References}

[1] Y. Huo, D. Yang, L. Ma, H. Wang, W. Ding, and S. Yang, "Oblique lumbar interbody fusion with stand-alone cages for the treatment of degenerative lumbar spondylolisthesis: a retrospective study with 1-year follow-up," Pain Research and Management, vol. 2020, Article ID 9016219, 6 pages, 2020.

[2] S. Yang, F. Zhang, J. Ma, and W. Ding, "Intervertebral disc ageing and degeneration: the antiapoptotic effect of oestrogen," Ageing Research Reviews, vol. 57, Article ID 100978, 2020.

[3] H. Wang, Z. Li, Y. Huo et al., "17 $\beta$-Estradiol alleviates intervertebral disc degeneration by inhibiting NF- $\kappa \mathrm{B}$ signal pathway," Life Sciences, vol. 284, Article ID 119874, 2021.

[4] T. J. Aalto, V. Leinonen, A. Herno et al., "Postoperative rehabilitation does not improve functional outcome in lumbar spinal stenosis: a prospective study with 2-year postoperative follow-up," European Spine Journal, and the European Section of the Cervical Spine Research Society, vol. 20, no. 8, pp. 1331-1340, 2011.

[5] A. F. Mannion, R. Denzler, J. Dvorak, M. Müntener, and D. Grob, "A randomised controlled trial of post-operative rehabilitation after surgical decompression of the lumbar spine," European Spine Journal, vol. 16, no. 8, pp. 1101-1117, 2007.

[6] R. M. A. van Erp, J. Jelsma, I. P. J. Huijnen, M. Lundberg, P. C. Willems, and R. J. E. M. Smeets, "Spinal surgeons' opinions on pre- and postoperative rehabilitation in patients undergoing lumbar spinal fusion surgery," Spine, vol. 43, no. 10, pp. 713-719, 2018.

[7] K.-å. Jansson, G. Németh, F. Granath, B. Jönsson, and P. Blomqvist, "Health-related quality of life (EQ-5D) before and one year after surgery for lumbar spinal stenosis," Journal of Bone and Joint Surgery British Volume, vol. 91-B, no. 2, pp. 210-216, 2009.

[8] K.-å. Jansson, G. Németh, F. Granath, B. Jönsson, and P. Blomqvist, "Health-related quality of life in patients before and after surgery for a herniated lumbar disc," Journal of Bone and Joint Surgery British Volume, vol. 87-B, no. 7, pp. 959964, 2005.

[9] A. F. Mannion, R. Denzler, J. Dvorak, and D. Grob, "Five-year outcome of surgical decompression of the lumbar spine without fusion," European Spine Journal, vol. 19, no. 11, pp. 1883-1891, 2010.

[10] B. I. Martin, S. K. Mirza, B. A. Comstock, D. T. Gray, W. Kreuter, and R. A. Deyo, "Reoperation rates following lumbar spine surgery and the influence of spinal fusion procedures," Spine, vol. 32, no. 3, pp. 382-387, 2007.

[11] T. Oosterhuis, L. O. Costa, C. G. Maher, H. C. de Vet, M. W. van Tulder, and R. W. Ostelo, "Rehabilitation after lumbar disc surgery," Cochrane Database of Systematic Reviews, vol. 2014, no. 3, Article ID CD003007, 2014.

[12] R. W. J. G. Ostelo, L. O. Pena Costa, C. G. Maher, H. C. W. de Vet, and M. W. van Tulder, "Rehabilitation after lumbar disc surgery," Spine, vol. 34, no. 17, pp. 1839-1848, 2009.

[13] R. W. Ostelo, L. O. P. Costa, C. G. Maher, H. C. de Vet, and M. W. van Tulder, "Rehabilitation after lumbar disc surgery," Cochrane Database of Systematic Reviews, vol. 8, no. 4, Article ID CD003007, 2008.

[14] S.-D. Yang, S.-H. Ning, L.-H. Zhang, Y.-Z. Zhang, W.-Y. Ding, and D.-L. Yang, "The effect of lower limb rehabilitation gymnastics on postoperative rehabilitation in elderly patients with femoral shaft fracture," Medicine, vol. 95, no. 33, Article ID e4548, 2016.

[15] S.-K. Liu, Y.-L. Song, W.-Y. Ding, D.-L. Yang, L. Ma, and S.-D. Yang, "The effect of systematic lower-limb rehabilitation training in elderly patients undergoing lumbar fusion surgery: a retrospective study," Oncotarget, vol. 8, no. 68, pp. 112720-112726, 2017.

[16] H. Wang, Y. Huo, Y. Zhao et al., "Clinical rehabilitation effect of postoperative lower-limb training on the patients undergoing OLIF surgery: a retrospective study," Pain Research and Management, vol. 2020, Article ID 1065202, 6 pages, 2020.

[17] M. S. Park, C.-N. Kang, W.-S. Lee et al., "A comparative study of the efficacy of NAXOZOL compared to celecoxib in patients with osteoarthritis," PLoS One, vol. 15, no. 1, Article ID e0226184, 2020.

[18] H. Yuan, Q.-Y. Chang, J. Chen et al., "A retrospective analysis of the effects of different analgesics on the pain of patients with traumatic thoracolumbar fractures in the peri-treatment period," Journal of Orthopaedic Surgery and Research, vol. 16, no. 1, Article ID 268, 2021. 
[19] M. Haffner, A. M. Saiz Jr., R. Nathe et al., "Preoperative multimodal analgesia decreases 24-hour postoperative narcotic consumption in elective spinal fusion patients," The Spine Journal, vol. 19, no. 11, pp. 1753-1763, 2019.

[20] P. R. Nielsen, L. D. Jørgensen, B. Dahl, T. Pedersen, and H. Tønnesen, "Prehabilitation and early rehabilitation after spinal surgery: randomized clinical trial," Clinical Rehabilitation, vol. 24, no. 2, pp. 137-148, 2010.

[21] N. Canbulat, M. Sasani, Y. Ataker et al., "A rehabilitation protocol for patients with lumbar degenerative disk disease treated with lumbar total disk replacement," Archives of Physical Medicine and Rehabilitation, vol. 92, no. 4, pp. 670-676, 2011.

[22] A. H. McGregor, C. J. Doré, T. P. Morris, S. Morris, and K. Jamrozik, "ISSLS prize winner," Spine, vol. 36, no. 21, pp. 1711-1720, 2011.

[23] O. Ilves, A. Häkkinen, J. Dekker et al., "Quality of life and disability: can they be improved by active postoperative rehabilitation after spinal fusion surgery in patients with spondylolisthesis? a randomised controlled trial with 12month follow-up," European Spine Journal, vol. 26, no. 3, pp. 777-784, 2017. 